\title{
Exosomes derived from human umbilical cord blood mesenchymal stem cells stimulate regenerative wound healing via transforming growth factor- $\beta$ receptor inhibition
}

Yan Zhang ${ }^{1,2}$, Yingjin Pan ${ }^{3}$, Yanhong Liu ${ }^{4}$, Xiheng Li ${ }^{1,2}$, Liang Tang ${ }^{1,2}$, Mengna Duan ${ }^{1 *}$, Jiang Li ${ }^{1,5^{*}}$ and Guokun Zhang ${ }^{6,7^{*}}$ (i)

\begin{abstract}
Background: Scar formation is a common consequence of skin wound healing, and no effective treatment exists. Umbilical cord blood mesenchymal stem cells (UCB-MSCs) can improve wound healing; however, the role of UCBMSCs remains unclear and whether they can ameliorate scar formation has not been fully elucidated.

Methods: To determine the function of UCB-MSCs, we examined and compared the therapeutic effects of UCBMSCs and UCB-MSC-derived exosomes (UCB-MSC-exo) on skin healing in rats. Moreover, UCB-MSC-exo-specific miRNAs were identified and their effects in inhibiting the human dermal fibroblast (HDF) differentiation into myofibroblasts were investigated.
\end{abstract}

Results: Both UCB-MSCs and UCB-MSC-exo accelerated wound closure; reduced scar formation; improved the regeneration of skin appendages, nerves, and vessels; and regulated the natural distribution of collagen fibers in wound healing. Additionally, UCB-MSC-exo suppressed the excessive formation of myofibroblasts and collagen I and increased the proliferation and migration of skin cells in vivo and in vitro. Functional analysis showed that UCBMSC-derived miRNAs were closely related to the transforming growth factor- $\beta$ (TGF- $\beta$ ) signaling pathway, which could induce myofibroblast differentiation. We identified abundant miRNAs that were highly expressed in UCBMSC-exo. miR-21-5p and miR-125b-5p were predicted to contribute to TGF- $\beta$ receptor type II (TGFBR2) and TGF- $\beta$ receptor type I (TGFBR1) inhibition, respectively. Using miRNA mimics, we found that miR-21-5p and miR-125b-5p were critical for anti-myofibroblast differentiation in the TGF- $\beta 1$-induced HDF.

\footnotetext{
*Correspondence: dmnmengna@jlu.edu.cn; ljiang@gzhmu.edu.cn; zhangguokun@hotmail.com

'Hospital of Stomatology, Jilin University, 1500 Qinghua Rd., Changchun, Jilin 130021, China

${ }^{6}$ Institute of Antler Science and Product Technology, Changchun Sci-Tech University, 1345 Pudong Rd., Changchun, Jilin 130600, China

Full list of author information is available at the end of the article
}

(c) The Author(s). 2021 Open Access This article is licensed under a Creative Commons Attribution 4.0 International License, which permits use, sharing, adaptation, distribution and reproduction in any medium or format, as long as you give appropriate credit to the original author(s) and the source, provide a link to the Creative Commons licence, and indicate if changes were made. The images or other third party material in this article are included in the article's Creative Commons licence, unless indicated otherwise in a credit line to the material. If material is not included in the article's Creative Commons licence and your intended use is not permitted by statutory regulation or exceeds the permitted use, you will need to obtain permission directly from the copyright holder. To view a copy of this licence, visit http://creativecommons.org/licenses/by/4.0/. The Creative Commons Public Domain Dedication waiver (http://creativecommons.org/publicdomain/zero/1.0/) applies to the data made available in this article, unless otherwise stated in a credit line to the data. 
Conclusion: The effect of UCB-MSCs in stimulating regenerative wound healing might be achieved through exosomes, which can be, in part, through miR-21-5p- and miR-125b-5p-mediated TGF- $\beta$ receptor inhibition, suggesting that UCB-MSC-exo might represent a novel strategy to prevent scar formation during wound healing.

Keywords: Cord blood stem cell transplantation, Exosomes, MicroRNAs, Myofibroblasts, Regeneration, Transforming growth factor beta, Wound healing

\section{Background}

Scar formation is a general consequence of wound healing after skin injury in adults [1-3], leading to psychological disorders and physical deformities. As there has not been an effective treatment until now, preventing or reducing scar formation is a significant problem in regenerative esthetics that urgently needs to be addressed. Pathogenesis of scar formation is assumed to occur via the recruitment and maintained differentiation of myofibroblasts, leading to excessive deposition of connective tissue (mainly collagen) [3, 4]. The differentiation of dermal fibroblasts in situ is the primary source of myofibroblasts, usually initiated by the TGF- $\beta$ signaling pathway $[4,5]$. In the process of wound healing, activated fibroblast proliferation and macrophage infiltration produce excess TGF- $\beta[4,5]$. TGF- $\beta$ binds to the TGF- $\beta$ receptor (TGFBR) of fibroblasts, thus activating downstream signaling of TGF- $\beta$ to induce myofibroblast differentiation, which further leads to the expression and extension of collagen fibers $[2,3,6,7]$. In this regard, interfering with the TGF- $\beta$ signaling pathway might inhibit myofibroblast differentiation from reducing scar formation.

Of all available treatments for the anti-scar formation and pro-ideal regeneration of skin appendages, mesenchymal stem cell (MSC) infusion has been considered a promising alternative strategy in numerous trials and clinical practices in the past few decades [8-10]. MSCs have several advantages that are essential for tissue repair, including relatively easy expansion in vitro, migration to the injured site, and differentiation into specific cell types required for tissue repair [9, 11-13]. Among the primary MSC sources that might be used for wound healing and regeneration of injured skin are adultderived MSCs [9], fetal-derived MSCs [14], embryonic stem cells (ESCs) [15], and induced pluripotent stem cells [16]. Human umbilical cord blood-derived MSCs (UCB-MSCs) have been used to improve wound healing, and it has been reported that they could accelerate wound closure of diabetic wounds and promote the expression of anti-scarring factors in mechanical wounds, as well as stimulate the rejuvenation of human skin [1721]. Compared with adult-derived MSCs, UCB-MSCs exhibit apparent advantages, including betterdocumented self-renewal, more multipotent differentiation properties, and lower immunogenicity [22, 23]. Compared with ESCs, UCB-MSCs are more easily obtained, as there are fewer related ethical issues [23]. However, the definite role of UCB-MSCs in vivo and their repair mechanisms in alleviating scar formation have not yet been fully elucidated.

The effect of MSCs in stem cell-based therapy is mainly via its secretion of paracrine pro-regenerative factors, including exosomes [9, 10, 24, 25]. Exosomes carry various cargo molecules, such as functional proteins, miRNAs, and signal lipids [26, 27], which can mediate cell-to-cell communication by initiating a series of biological responses in recipient cells. After these exosomes are secreted, the recipient cells take up these exosomes through phagocytosis/endocytosis or fusion, thereby receiving cargo.

In this study, we evaluated the effects of UCB-MSCs and UCB-MSC-derived exosomes (UCB-MSC-exo) on scar formation during wound healing in rats. We revealed that the therapeutic capacity of UCB-MSCs on wound healing could be achieved by exosomes. Additionally, we identified two specific miRNAs carried by UCB-MSC-exo as essential factors that inhibit fibroblast differentiation into myofibroblasts via inhibition of TGF$\beta$ receptor activity. The results suggest that UCB-MSCexo might represent a novel strategy to prevent scar formation and improve skin appendage regeneration during wound healing in the clinic.

\section{Materials and methods \\ Cell culture and characterization}

Human UCB-MSCs were a gift from the Bethune First Hospital of Jilin University and were used with informed consent. The Medical Ethics Committee of Hospital of Stomatology of Jilin University approved the research about UCB-MSCs (2020[42]). The protocol for UCBMSC isolation was processed as previously described [18]; briefly, umbilical cord blood was collected from delivered placentas using $50 \mathrm{ml}$ syringes (contained $1000 \mathrm{U}$ of heparin) and then diluted with PBS. Mononuclear cells were isolated by density gradient centrifugation using Ficoll-Hypaque-Plus solution (Solarbio, China). Freshly isolated mononuclear cells were suspended in Dulbecco's modified Eagle's medium (Hyclone, USA) containing $10 \%$ fetal bovine serum (Hyclone, USA) and $1 \%$ penicillin/streptomycin (Biological Industries, Israel) and incubated at $37^{\circ} \mathrm{C}$ in $5 \% \mathrm{CO}_{2}$. We continued the cultures for another 7 days after fibroblast-like cells 
appeared on the bottom of the flasks. Then, these fibroblast-like cells were collected and expanded. The second to fifth passage cells were used for experiments.

UCB-MSCs were characterized by surface marker profiling (CD73, CD90, and CD105) via flow cytometry (FCM) and immunofluorescence (IF) staining, as previously described [28]. For FCM, the cell suspension was stained with primary antibodies, washed three times with PBS, stained with secondary antibodies, washed three times with $\mathrm{PBS}$, and quantitatively analyzed using a BD FACSCelesta (BD Biosciences, USA). Table S1 lists the antibodies used. The process of IF staining is described in the following section. The multipotency of UCBMSCs was detected via inducing adipogenic (Oil Red-O staining), osteogenic (Alizarin Red S staining), and chondrogenic (Alizarin Blue staining) differentiation.

The human dermal fibroblasts (HDFs) and epidermal stem cells (EPSCs) were purchased commercially (Dingguo, Beijing, China). The cell lines used in this study were all cultured in DMEM containing 10\% fetal bovine serum and $1 \%$ penicillin/streptomycin and incubated at $37^{\circ} \mathrm{C}$ in $5 \% \mathrm{CO}_{2}$.

\section{Exosome preparation}

UCB-MSCs (passage 4 to passage 8) were seeded into T175-cell culture flasks and allowed to reach $70 \%$ to $80 \%$ confluence. The media was then replaced by a serum-free medium (Hyclone, USA), and the cells were cultured for another $48 \mathrm{~h}$. Next, the conditioned medium was collected and centrifuged to remove the dead cells and debris, and then, collected exosomes were washed three times and stored at $-80^{\circ} \mathrm{C}$ (Fig. 1A). UCB-MSCs were characterized using a NanoSight NS300 (Malvern Instruments, UK), transmission electron microscopy, and Western blot of CD9 and TSG101 [9].

\section{In vivo wound generation and UCB-MSC-exo treatment}

Female SD rats (6-8 weeks old; $220-250 \mathrm{~g}$ ) were purchased from Liaoning Changsheng Biotechnology Co., Ltd. (Benxi, China). The animal experiment protocol and procedure were approved by the Animal Experiment Ethics Committee of Jilin University (approval no.: SY202008010). Rats were randomly assigned to UCBMSC, UCB-MSC-exo, or control groups (10 rats/group). All rats were anesthetized by $3 \%$ pentobarbital sodium $(30 \mathrm{mg} / \mathrm{kg})$ before surgery. A $12-\mathrm{mm}$ diameter excisional wound was created on the dorsal region under sterile surgical conditions. The rats were treated with UCBMSCs $\left(2 \times 10^{6}\right.$ cells $)$ or UCB-MSC-exo $(20 \mu \mathrm{g})$ by tail vein injection $(100 \mu \mathrm{l})$. Rats in the control group were injected with an equivalent volume of PBS. The above treatment was conducted weekly. The extent of wound healing was photographed every week. Rats were sacrificed 2 or 4 weeks after surgery, and the healing skin tissue was collected for histological and quantitative real-time polymerase chain reaction (qRT-PCR) analysis.

\section{Cell counting kit-8 (CCK8) assay}

EPSCs or HDFs were seeded into 96-well plates at a density of 10,000 cells/well $(100 \mu \mathrm{l})$ and cultured for 24 $\mathrm{h}$, and then UCB-MSC-exo $(25 \mathrm{ng} / \mathrm{ml})$ were added to the culture for another $24 \mathrm{~h}$. To each well was added $10 \mu \mathrm{l}$ CCK-8 solution (MedChemExpress, Shanghai, China), and then cultures were incubated for $4 \mathrm{~h}$ in an incubator. Absorbance was measured at $450 \mathrm{~nm}$ using a microplate reader.

\section{Cell scratch assay}

EPSCs or HDFs were seeded into 24-well plates at a density of 50,000 cells/well $(500 \mu \mathrm{l})$ and cultured for 24 $\mathrm{h}$. The cell monolayer was scraped along a straight line with a P-200 pipette tip to form a "scratch". The debris was removed, the edge of the scratch was smoothed by washing the cells with PBS, and then the medium was replaced with $200 \mu \mathrm{l}$ of medium containing $25 \mathrm{ng} / \mathrm{ml}$ UCB-MSC-exo.

\section{In vitro myofibroblast differentiation induction and UCB-} MSC-exo/miRNA-mimic treatment

HDFs were seeded into 24-well plates at a density of 50,000 cells/well $(500 \mu \mathrm{l})$ combined with TGF- $\beta 1$ ( $25 \mathrm{ng} /$ $\mathrm{ml}$ ) and cultured for $48 \mathrm{~h}$ to induce myofibroblast differentiation. At the same time, UCB-MSC-exo $(25 \mathrm{ng} / \mathrm{ml})$ or miRNA mimics $(50 \mathrm{nM})$ were added to each well. The expression of Collagen I, $\alpha$-SMA, TGFBR1, and TGFBR2 was measured by IF staining and qRT-PCR analysis.

\section{Histopathological analysis}

Healing skin was fixed with $4 \%$ paraformaldehyde solution, dehydrated by graded ethanol, and embedded in paraffin. The sections were cut into slices and stained with hematoxylin and eosin (H\&E) or Masson and photographed under a microscope (Precipoint M8, Germany). The numbers of skin appendages and the levels of collagen fibers (blue) and myofibers (red) in the wounded area were calculated with Image-Pro Plus software. For IF staining, rehydrated antigen-repaired paraffin sections or fixed cells were incubated with primary antibodies and conjugated secondary antibodies, stained with 4',6-diamidino-2-phenylindole dihydrochloride (DAPI) (Beyotime, China) and photographed under a microscope (VOS M5000, USA). Table S1 lists the antibodies used.

\section{qRT-PCR}

Total RNA was isolated from healing skin, HDFs, and UCB-MSC-exo using TRIzol reagent (Sangon Biotech 
A

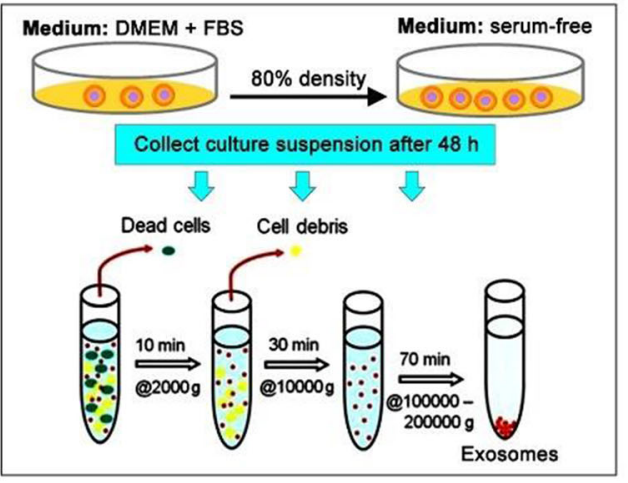

B

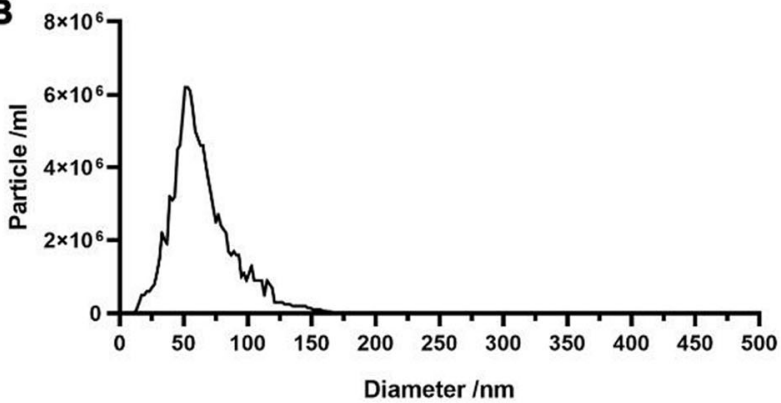

C

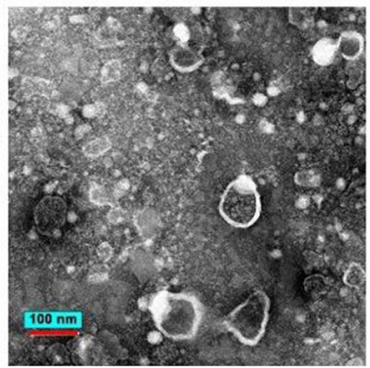

E

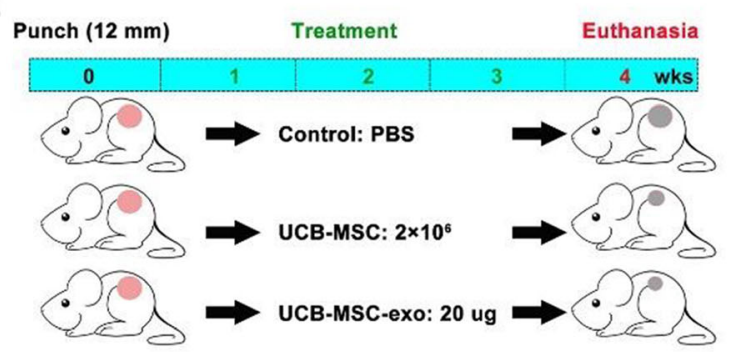

$\mathbf{F}$

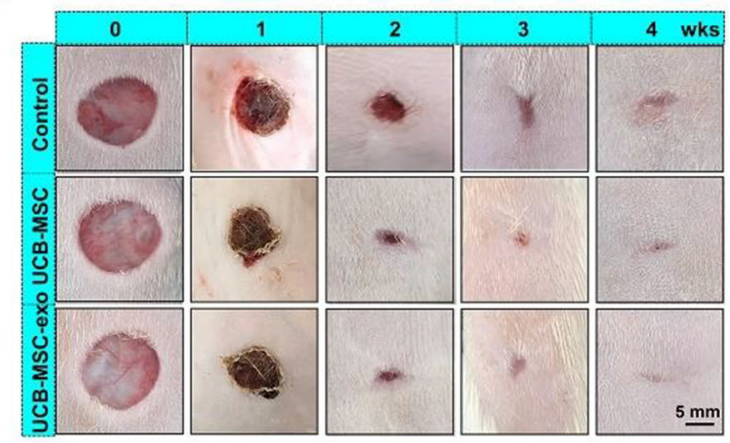

G

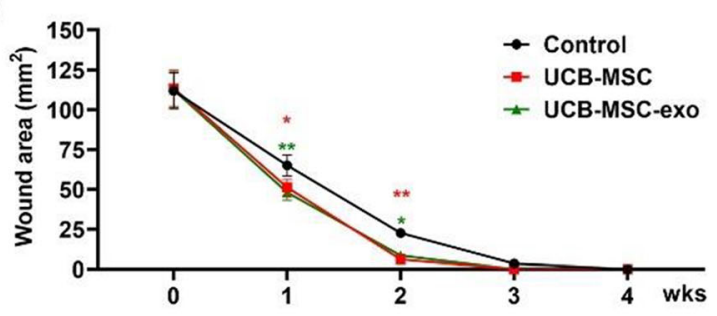

Fig. 1 UCB-MSC-exo accelerate wound closure and suppress scar formation in full-thickness skin-wounded rats. A Schematic representation of UCB-MSC-exo preparation. B UCB-MSC-exo particle size was measured using NanoSight. C Morphological characteristics of UCB-MSC-exo were measured using transmission electron microscopy (scale bar $=100 \mathrm{~nm}$ ). D Exosomal markers of UCB-MSC-exo were measured via western blot. $\mathbf{E}$ Schematic representation of animal experiments. The full-thickness excisional wound ( $12 \mathrm{~mm}$ diameter) was created on the dorsal region. The rats were then administered by tail vein injection every week: PBS (control group), UCM-MSCs $\left(2 \times 10^{6}\right.$ cells), or UCB-MSC-exo $(20 \mu \mathrm{gg})$. F Morphological changes during wound healing; scale bar $=5 \mathrm{~mm}$. G Changes in the wound area during wound healing. ${ }^{*} p<0.05 ;{ }^{* *} p<0.01$; means $\pm \mathrm{SD} ; n=5$

Co., Ltd., Shanghai, China). cDNA was synthesized from total RNA using the cDNA Synthesis Kit (Takara, Japan). qRT-PCR analysis was performed using SYBR Green Master (Roche, Switzerland) in an ABI 9700 Detection System (Thermo Fisher Scientific, MA, USA). GAPDH mRNA was used as an internal control. qRT-PCR for miRNA was performed using the Bulge-Loop ${ }^{\text {TM }}$ miRNA qRT-PCR Starter Kit (Ribobio, China) according to the manufacturer's instructions. U6 small RNA was used as an internal control. Table S2 lists the primers used. All experiments were repeated in triplicate.

\section{Statistical analysis}

All quantitative data are shown as means $\pm \operatorname{SD}(n \geq 3)$. Statistical analysis was conducted using Graphpad Prism software, and significant differences were evaluated using a one-way analysis of variance; $p<0.05$ was considered statistically significant.

\section{Results}

UCB-MSC-exo accelerate wound closure and suppresses scar formation in full-thickness skin-wounded rats

UCB-MSCs expressed the putative mesenchymal markers CD73, CD90, and CD105 and could be induced 
to adipogenic, osteogenic, and chondrogenic differentiation (Figure S1). These results confirmed the phenotypic characterization of cells as MSCs. UCB-MSC-exo were prepared from UCB-MSC-conditioned medium by gradient centrifugation (Fig. 1A). It was characterized by a discoid morphology (Fig. 1B) with a diameter of 30$150 \mathrm{~nm}$ (Fig. 1C) determined by transmission electron microscopy and NanoSight. The result of Western blot showed that UCB-MSC-exo expressed exosomal markers CD9 and TSG101 (Fig. 1D).

The therapeutic potentials of UCB-MSCs and UCBMSC-exo were compared in full-thickness skin-wounded rats (Fig. 1E). As expected, there were no significant differences in wound closure and scar area between the UCB-MSC-exo and UCB-MSC groups; however, UCBMSC-exo and UCB-MSC significantly accelerated wound closure and smoothed wound edges, resulting in a smaller scar size than in the control group (Fig. 1F, G). These results suggest that UCB-MSCs accelerate wound closure and suppress scar formation, which may function via UCB-MSC-exo.

\section{UCB-MSC-exo improve regeneration and regulates collagen distribution in wound healing of rats}

Pathological analysis via $\mathrm{H} \& \mathrm{E}$ staining showed that healing skin in the UCB-MSC and the UCB-MSC-exo groups exhibited more appendages than that in the control group. Interestingly, the skin in the UCB-MSC group exhibited significantly $(p<0.01)$ more appendages than that in the UCB-MSC-exo group after 2 weeks of treatment; however, there were no significant differences after 4 weeks of treatment (Fig. 2A-C). There were also significantly $(p<0.05)$ fewer collagen fibers (blue) and myofibers (red) in both the UCB-MSC and the UCBMSC-exo groups than in the control group by Masson staining (Fig. 2A, C, D). Moreover, the ratios of collagen fibers to myofibers among the groups displayed different trends after 2 and 4 weeks of treatment (Fig. 2E); the ratios in the UCB-MSC and UCB-MSC-exo groups were lower than those of the control group after 2 weeks of treatment and higher after 4 weeks of treatment.

Furthermore, we evaluated the effects of UCB-MSCexo on nerve and vessel regeneration in healing skin. IF staining results showed that, after 2 or 4 weeks of treatment, the skin in the UCB-MSC-exo and UCBMSC groups exhibited significantly more vessels $\left(\mathrm{CD} 31^{+}\right)$and nerves $\left(\right.$nestin $\left.^{+}\right)$than that in the control group (Fig. 2F). These results suggest that UCB-MSCs and UCB-MSC-exo have similar effects on skin regeneration, promoting the regeneration of skin appendages, vessels, and nerves, decreasing fiber formation, and regulating the ratio of collagen fiber to myofiber in the wound healing process.
UCB-MSC-exo promote the proliferation and migration of skin cells in vivo and in vitro

To investigate the mechanism by which UCB-MSC-exo accelerate wound closure, we examined the effects of UCB-MSC-exo on the proliferation and migration of skin cells in vivo and in vitro (Fig. 3). The results showed that the cell division in healing skin in the UCB-MSCexo group was higher than that in the control group (Fig. 3A). Additionally, UCB-MSC-exo also promoted the proliferation and migration of EPSCs and HDFs in vitro (Fig. 3B-C). These results suggest that UCBMSC-exo accelerate wound closure, which can occur, in part, via the promotion of the proliferation and migration of skin cells during wound healing.

\section{UCB-MSC-exo suppress scar formation by inhibiting myofibroblast differentiation}

$\alpha$-SMA and collagen I are critical markers of myofibroblasts and scar tissue. The results showed that $\alpha$-SMA and collagen I were highly expressed in the healing skin; however, the expression levels were reduced by UCBMSC-exo treatment (Fig. 4A-C). To further verify these results, HDFs were cultured in the presence of TGF- $\beta 1$ to induce myofibroblast differentiation in vitro, and UCB-MSC-exo $(25 \mathrm{ng} / \mathrm{ml})$ were used to intervene. Expectedly, UCB-MSC-exo treatment strongly inhibited the TGF- $\beta 1$-induced high expression of $\alpha$-SMA and collagen I (Fig. 4D-F). These results suggest that UCBMSC-exo may suppress scar formation by inhibiting myofibroblast differentiation.

\section{UCB-MSC-exo-derived miRNAs inhibit myofibroblast differentiation via targeting TGF- $\beta$ receptor}

miRNAs are the main components of exosomal functional RNA $[9,10]$. We analyzed the miRNAs in UCBMSCs as reported by Meng et al. [28] (Fig. 5A). To further reveal the possible roles of these miRNAs, we used Gene Ontology analysis to predict their function. We demonstrated that these miRNAs were positively correlated with the TGF- $\beta$ signaling pathway (Fig. 5B). Generally, MSC- and their exosome-derived miRNAs were highly correlated; thus, we detected the levels of miRNAs in UCB-MSC-exo, which were similarly highly expressed in UCB-MSCs. We found that miR-21-5p, miR-125b-5p, miR-100-5p, miR-31-5p, and let-7a-5p were highly expressed in UCB-MSC-exo (Fig. 5C). Moreover, we predicted the target genes of these miRNAs using TargetScan (http://www.targetscan.org/) and found that miR-21-5p and miR-125b-5p were predicted to target TGFBR2 and TGFBR1 mRNAs directly, respectively (Fig. 6A).

miRNA mimics were added into the HDF (+TGF- $\beta 1)$ culture system to determine whether or not these miRNAs could affect the expression of TGFBR2 and 

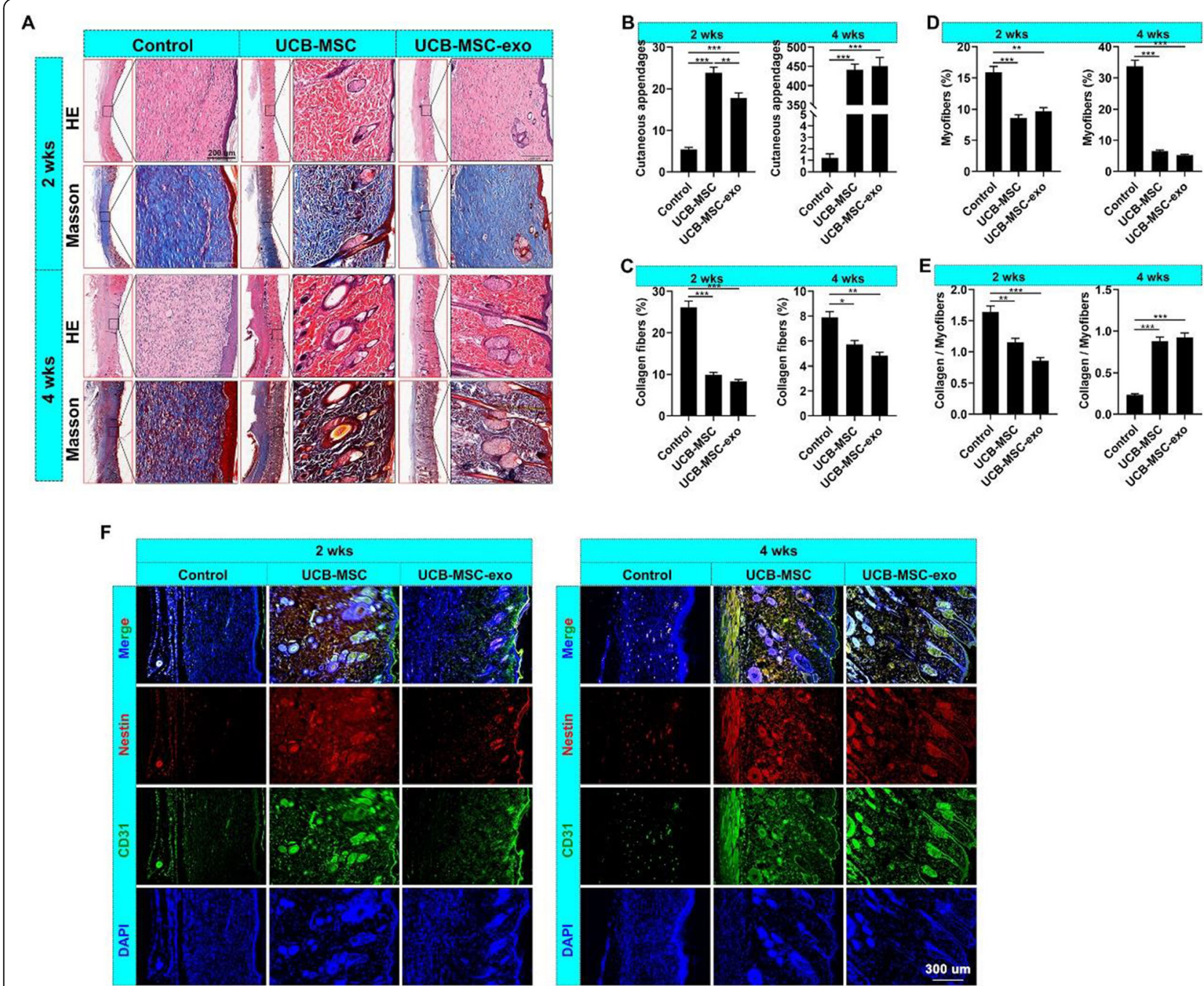

Fig. 2 UCB-MSC-exo improve the regenerative wound healing in rats. A H\&E and Masson staining of the healing skin; scale bar $=200 \mu \mathrm{m}$. B Number of skin appendages (hair follicles and sebaceous glands) in the healing skin. C-E Levels of collagen fibers (blue), myofibers (red) and the ratio of collagen fibers to myofibers in the healing skin, per Masson staining. $\mathbf{F}$ IF staining of Nestin (nerves) and CD31 (vessels) in the healing skin; scale bar $=300 \mu \mathrm{m} .{ }^{*} p<0.05 ;{ }^{* *} p<0.01 ;{ }^{* * *} p<0.001$; means \pm SD; $n=3$

TGFBR1 and thus verify the roles of miR-21-5p and miR-125b-5p in UCB-MSC-exo function. The results showed that miR-21-5p and miR-125b-5p significantly inhibited TGFBR 2 and TGFBR1, respectively, and decreased the expression of $\alpha$-SMA (Fig. 6B-G). Moreover, the expression levels of TGFBR2 and TGFBR1 in the healing skin of rats were also reduced by UCB-MSC-exo treatment (Fig. 7). These results suggest that UCB-MSCexo suppress myofibroblast differentiation, which can be, in part, via the expression of specific miRNAs miR-21$5 \mathrm{p}$, targeting TGFBR2, and miR-125b-5p, targeting TGFBR1.

\section{Discussion}

Adult wound healing generally comprises scar repair, which may be the result of excessive myofibroblast formation [1, 2]. MSCs are a promising technique to accelerate wound closure and limit scarring in wound healing [9, 14-16]. UCB-MSCs have been used to accelerate the closure of diabetic wounds and have been reported to promote the expression of anti-scarring factors [17-21]. However, few attempts have been made to study the effects of UCB-MSCs on scar formation. More and more studies have shown that MSCs play a role mainly through immune regulation and paracrine processes, rather than directly differentiating $[9,10,14,29]$. UCB-MSC-exo are the primary source of UCB-MSC paracrine factors; it remains unclear whether these are the main effectors of UCB-MSC function, especially as the roles of UCB-MSC-exo-derived "cargo" is unknown. For the first time, the present study has demonstrated that UCB-MSCs accelerate wound closure and suppress 


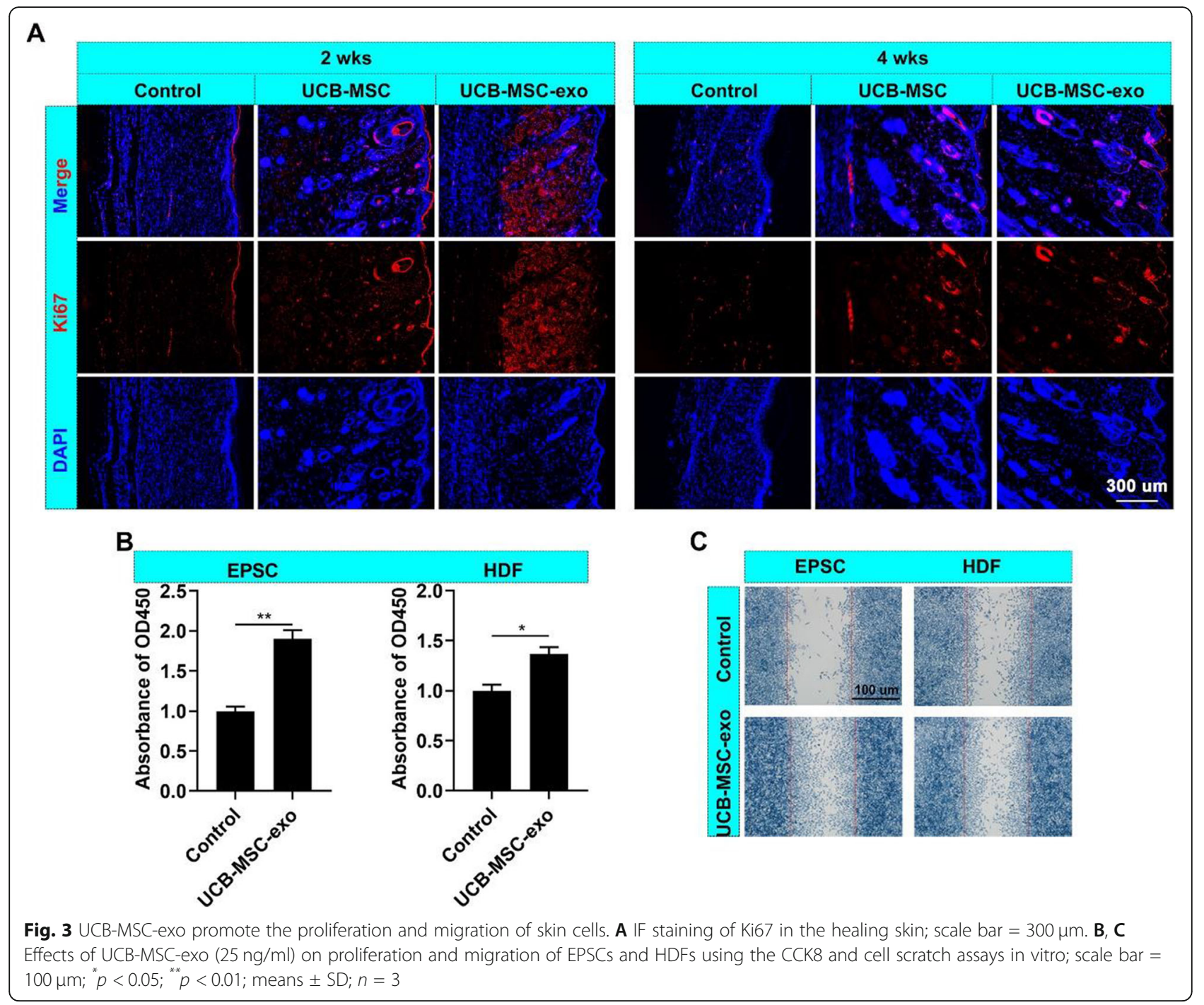

scar formation in wound healing, which can be, in part, via UCB-MSC-exo. We also identified two specific miRNAs carried by UCB-MSC-exo that could inhibit the signaling activities of TGF- $\beta$ receptors, thereby inhibiting myofibroblast differentiation. To a certain extent, our research proposes a new strategy for the clinical application of UCB-MSCs to prevent scar formation.

In wound healing, MSC-based therapy is a promising strategy to enhance re-epithelialization, inhibit fibrotic remodeling, promote regeneration of skin appendages, increase angiogenesis, stimulate endogenous stem cell recruitment, and modulate inflammation $[9,10,14-16$, 29]. Additionally, UCB-MSCs might represent a more promising option that has the potential to overcome several limitations, including greater expansion capacity with differentiation potential, lower immunogenicity compared with adult-derived MSCs, and easier obtainment compared to ESCs, due to related ethical issues $[22,23]$.
UCB-MSCs have been used to enhance reepithelialization and improve the quality of wound healing. Jae-A Jung et al. [19] and N Çil et al. [20] respectively reported that UCB-MSCs promote diabetic wound healing by increasing collagen synthesis and neovascularization. Moreover, Hanako Doi et al. [18] found that UCB-MSCs express lower levels of IL-1 proinflammatory cytokines and higher levels of MMP1 and PLAU ECM degradation enzymes than Wharton's jelly MSCs, suggesting that UCB-MSCs were more likely to favor scarless wound healing. However, they failed to significantly reduce scar formation following direct injection of UCB-MSCs into full-thickness skin-wounded nude mice [18]. In the present study, we evaluated the essential roles of UCB-MSCs in the wound healing of rats. We observed the phenomena of earlier wound closure, smaller scar formation, and more skin appendages, nerves, and vessels in healing skin in the UCB-MSC group compared with those in the control group. 


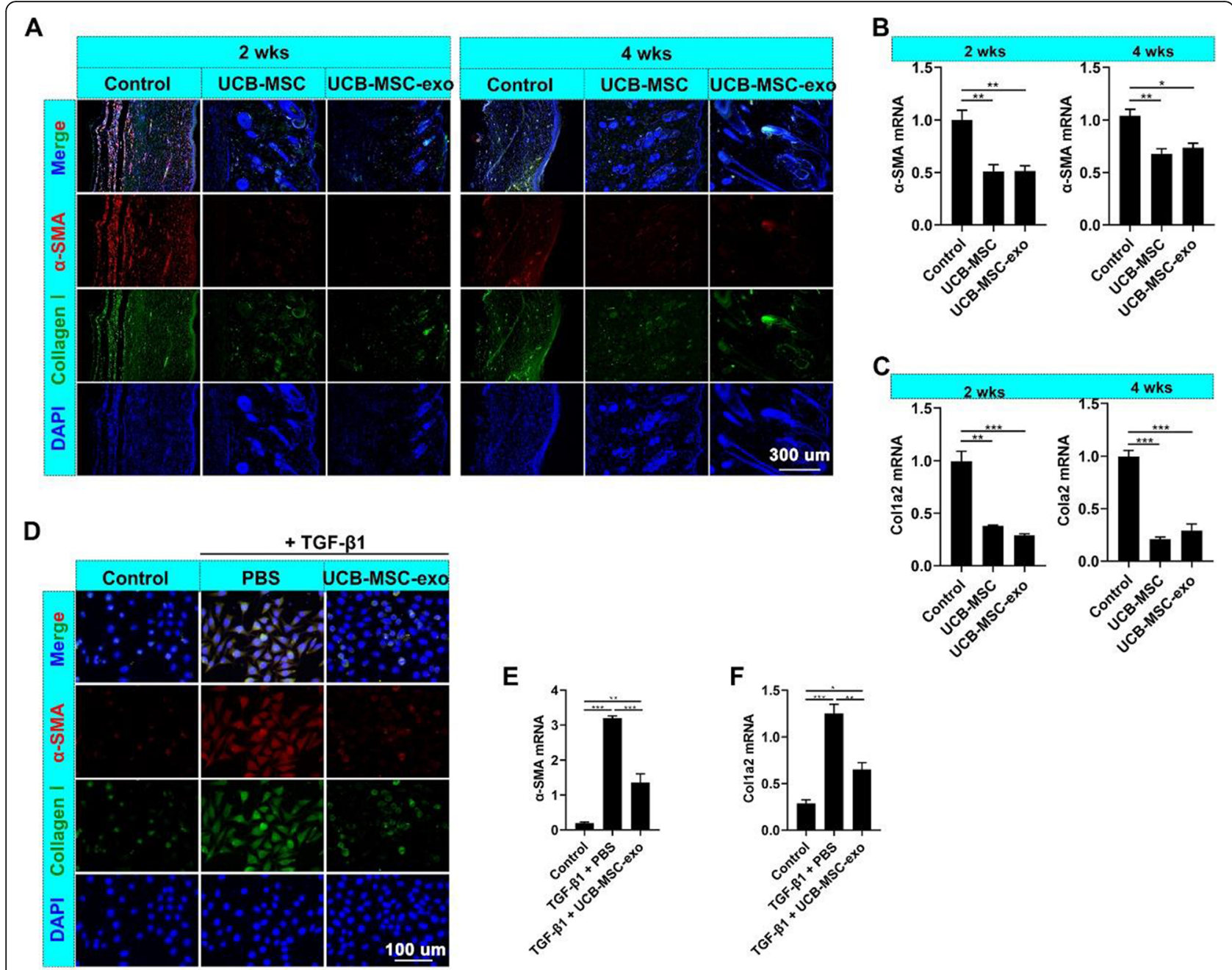

Fig. 4 UCB-MSC-exo suppress myofibroblast formation. A IF staining of a-SMA and collagen I in the wound healing skin; scale bar $=300 \mu \mathrm{m}$. B, C mRNA levels of $\alpha-S M A$ and Col1a2 in the wound healing skin. HDFs were cultured for $48 \mathrm{~h}$ with TGF- $\beta 1$ ( $25 \mathrm{ng} / \mathrm{ml})$ to induce myofibroblast differentiation. Some cells were also treated with UCB-MSC-exo $(25 \mathrm{ng} / \mathrm{ml})$. D-F IF staining and qRT-PCR analysis of a-SMA and collagen I in HDFs; scale bar $=100 \mu \mathrm{m} .{ }^{*} p<0.05 ;{ }^{* *} p<0.01 ;{ }^{* * *} p<0.001$; means \pm SD; $n=3$

Myofibroblast differentiation and scar formation were also inhibited by UCB-MSC treatment, as indicated by the decreased expressions of $\alpha$-SMA and collagen I in the healing skin of rats. Classically, $\alpha$-SMA is considered a marker of myofibroblast differentiation [30, 31], and collagen I is considered the main component of scar tissue $[2,3]$. The over-differentiation of myofibroblasts and the continuous expression of collagen I is a fundamental cause of scar formation [2,3]; thus, intervention is required during wound healing to prevent myofibroblast accumulation rather than taking remedial measures after scar formation. This study observed different in vivo experimental results from those observed by Hanako Doi et al. [18]. This might be due to differences in UCBMSC treatment methods (tail vein injection versus local injection around the wound), suggesting that the diseased microenvironment is not conducive to MSC survival and retention, limiting treatment efficacy. We also observed that UCB-MSCs could inhibit collagen deposition, which is counter to the results of Jae-A Jung et al. [19], perhaps due to the different animal wound models used (mechanical wounds versus diabetic wounds), as diabetic wound healing is different from mechanical wound healing, it may be in the early stage of healing for a long time. It is known that MSCs can regulate collagen remodeling to inhibit scar hyperplasia. In an early stage, MSCs promote collagen remodeling through the synthesis of types I and III, whereas they reduce scarring in the late stage by inhibiting collagen formation [32]. This may suggest that UCB-MSCs have a flexible targeting effect in different pathologies, which may increase the scope of its application.

It is currently believed that MSCs play a therapeutic role in vivo, mainly through paracrine signaling $[9,10$, 


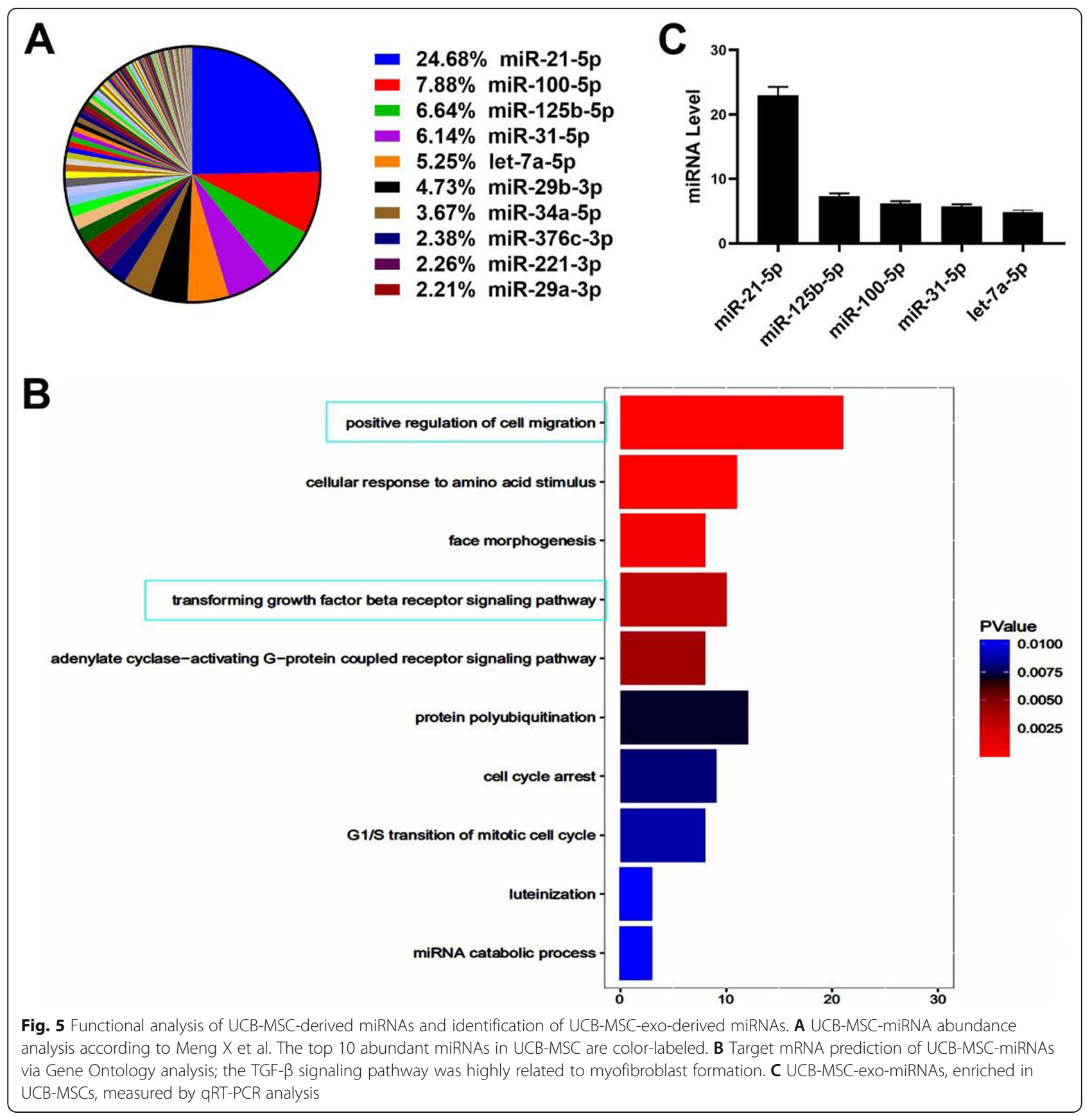

14, 29]. MSCs can release biologically active molecules to affect the proliferation, migration, and survival of receptor cells. Our recent studies have reported that EPSCs [9] and amniotic fluid stem cells [10] promote skin regeneration via secreted exosomes. In this study, we also evaluated the essential roles of UCB-MSC-exo in the wound healing of rats and found that the effect of UCB-MSC-exo was similar to that of UCB-MSCs, suggesting that the regenerative healing effect of UCB-MSC on wound healing may be achieved via exosome secretion. We tracked UCB-MSC in vivo and detected whether they exist on the wounded site 1 week and 2 weeks after transplantation. Interestingly, we found that UCB-MSC aggregation was detected at the wounded site 1 week after cell implantation. However, there are indeed very few UCB-MSCs after cell implantation after 2 weeks (data not shown), suggesting that few UCB-MSCs become permanently engrafted within the repaired tissue. Besides the regenerative repair of skin wounds to achieve scar-free healing, rapid wound closure is also critical because wound closure is essential to block external environmental interference. Although the 


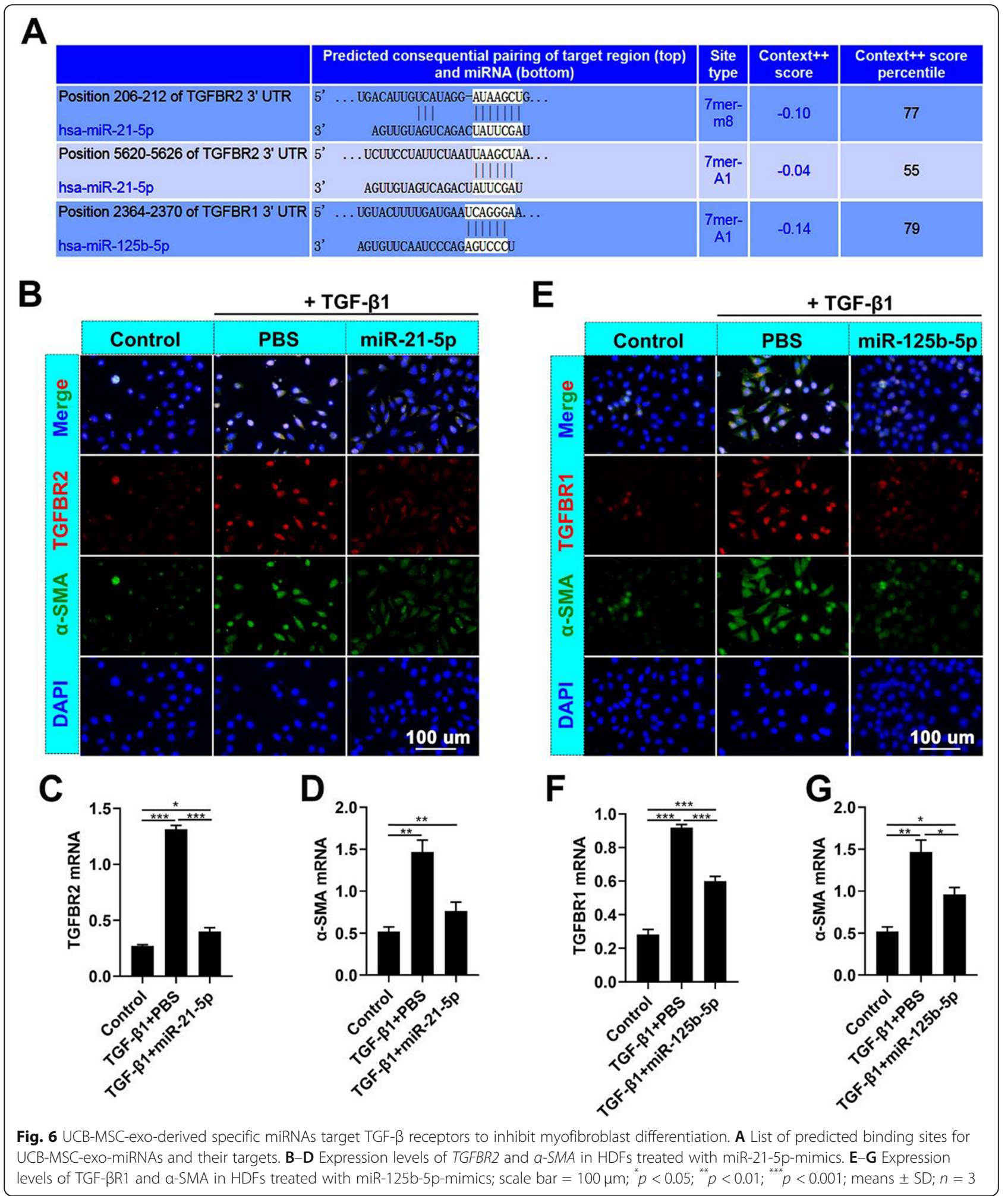

contractility of myofibroblasts is beneficial to wound closure in the early stage, the inhibition of UCB-MSCexo on myofibroblast differentiation does not necessarily lead to a slowdown in wound healing. We found that
UCB-MSCs can promote the proliferation and migration of skin cells, including EPSCs and HDFs, which may be essential for accelerating wound closure; this is similar to the results reported by Yoon-jin Kim et al. [19]. UCB- 


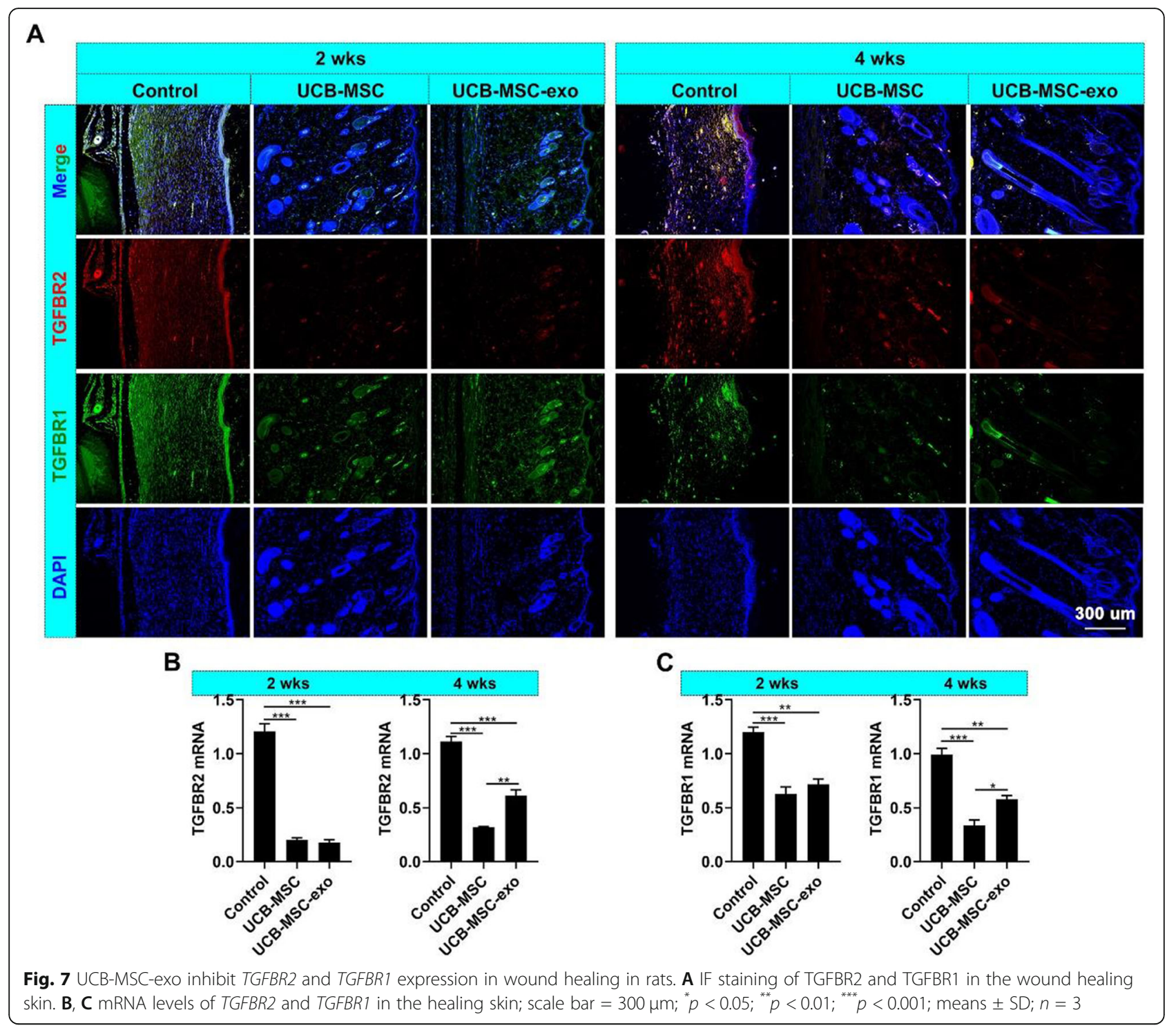

MSCs and UCB-MSC-exo have similar repair effects on wound healing; however, we believe that UCBMSC-exo may have other advantages, such as overcoming the immune and tumorigenic issues caused by allotransplantation.

Exosomes generally play a crucial role in cell communication, transmitting information to neighboring cells through their "cargo," including proteins, DNA, mRNAs and miRNAs [26, 27,33]. The therapeutic potential of exosomes depends on the composition of the "cargo" they carry [33]. It has been reported that miRNAs are generally abundant in exosomes [9, 10]; these miRNAs regulate various signaling pathways in the receptor cells, including cell trafficking, apoptosis, angiogenesis, and proteolysis via targeting transcription factors and genes [33]. We analyzed the miRNAs in UCB-MSCs as reported by Meng et al. [28] and found that these miRNAs were positively correlated with the TGF- $\beta$ signaling pathway. Generally, the MSC-derived miRNAs and their exosome-derived miRNAs are highly correlated, which is confirmed by our results. We found that the effect of anti-myofibroblast differentiation may result from a pair of TGF- $\beta$ receptor-targeting miRNAs, miR-21-5p, and miR-125b-5p. miR-125b-5p has been reported to suppress liver fibrosis in non-alcoholic fatty liver disease by inhibiting the RhoA signaling pathway [34]. In our study, miR-125b-5p also suppressed fibrosis scarring by inhibiting TGFBR1 expression. Additionally, miR-21-5p could inhibit TGFBR2 expression in the TGF- $\beta$ signaling pathway, thereby suppressing myofibroblast differentiation, in contrast to other reports, in which miR-21-5p promoted gastric cancer and kidney fibrosis by upregulating Smad7 in the TGF- $\beta$ signaling pathway $[35,36]$. Generally, miRNAs can target different mRNAs 


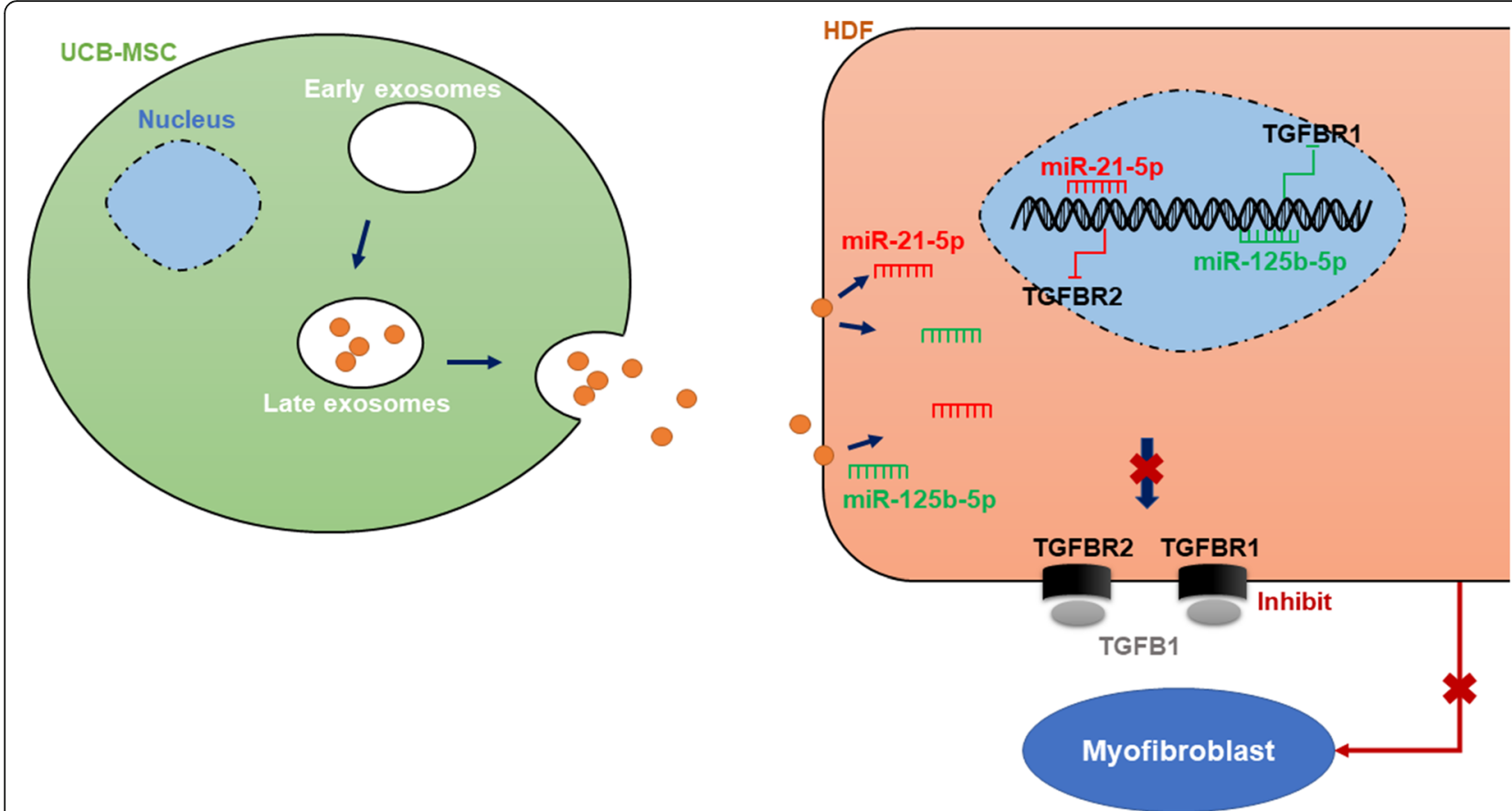

Fig. 8 UCB-MSC-exo improve regenerative wound healing and suppress scar formation by inhibiting the expression of TGF- $\beta$ receptors. When UCB-MSCs are transplanted into the skin wound, multiple vesicles bud from the plasma membrane, releasing the exosomes into the extracellular space where they then fuse with the plasma membrane of receptor cells. UCB-MSC-exo, carrying cargos of proteins, and RNAs, can travel to and be internalized by recipient cells (HDFs). Then, UCB-MSC-exo-derived specific miRNAs (such as miR-21-5p and miR-125b-5p) can regulate gene expression in HDFs. miR-21-5p targets TGFBR2, whereas miR-125b-5p targets TGFBR1, thereby suppressing myofibroblast differentiation, and ultimately reducing scar formation and promoting regenerative wound healing

simultaneously. Therefore, we believe that miR-21-5p may be a double-edged sword regulating TGF- $\beta$ signaling; its function may vary according to the cell state and the specific molecular network involved. Based on the above results, we suggest that UCB-MSC-exo miRNAs may be important regulators of the TGF- $\beta$ signaling pathway by inhibiting myofibroblast differentiation during wound healing in the skin.

\section{Conclusions}

The present study revealed that UCB-MSCs could stimulate regenerative wound healing via their exosomes. Through exosome-mediated intercellular transfer, miR21-5p and miR-125b-5p derived from UCB-MSC-exo inhibited TGFBR2 and TGFBR1, respectively, thereby inhibiting the TGF- $\beta$ signaling pathway to suppress myofibroblast differentiation (Fig. 8). As an alternative to cell therapy, UCB-MSC-exo might represent a novel strategy to prevent scar formation during wound healing in the clinic.

\section{Abbreviations}

a-SMA: a-Smooth muscle actin; CCK8: Cell counting kit; EPSC: Epidermal stem cells; FCM: Flow cytometry; H\&E: Hematoxylin and eosin; HDF: Human dermal fibroblasts; IF: Immunofluorescence staining; PBS: Phosphate-buffered saline; TGF- $\beta 1$ : Transforming growth factor- $\beta 1$; TGFBR1: TGF- $\beta$ receptor type I;
TGFBR2: TGF- $\beta$ receptor type Il; qRT-PCR: Quantitative real-time polymerase chain reaction; UCB-MSC: Umbilical cord blood mesenchymal stem cell; UCBMSC-exo: UCB-MSC-derived exosomes

\section{Supplementary Information}

The online version contains supplementary material available at https://doi. org/10.1186/s13287-021-02517-0.

Additional file 1: Figure S1. Phenotypical characterization of UCBMSCs. A FCM analysis of expression profiles of cell surface markers CD73, CD90, and CD105. B IF staining analysis expression profiles of cell surface markers CD73, CD90, and CD105; scale bar = 300 um. Table S1. Antibodis. Table S2. Primers.

Additional file 2. Gene ontology.

\section{Acknowledgements}

We thank Dr. Shengnan Wang and Dr. Jing Ren from the Changchun SciTech University for excellent technical assistance in bioinformatics and histological analysis.

\section{Authors' contributions}

$G Z$ and $J L$ designed and supervised the research, $Y Z$ and $M D$ performed the research and analyzed the data, YP and YL contributed to animal experiments, $\mathrm{XL}$ and LT contributed to histopathological experiments, and YZ and GZ wrote and edited the paper. All authors read and approved the final manuscript.

\section{Funding}

The study was supported by a grant from the Department of Science and Technology of Jilin Province (No.20200404174YY; No.20200201310JC) and a 
grant from the Science and Technology Project of Jilin Provincial Finance Department (No. jcsz2020304-19).

\section{Availability of data and materials}

The datasets used and/or analyzed during the present study are available from the corresponding author on reasonable request.

\section{Declarations}

\section{Ethics approval and consent to participate}

The animal experiments were approved by the Animal Experiment Ethics Committee of Jilin University (Approval NO. SY202008010). Human UCB-MSC experiments were used with informed consent and approved by the Medical Ethics Committee of Hospital of Stomatology of Jilin University (2020[42]).

\section{Consent for publication}

Not applicable.

\section{Competing interests}

The authors declare that they have no competing interests.

\begin{abstract}
Author details
${ }^{1}$ Hospital of Stomatology, Jilin University, 1500 Qinghua Rd., Changchun, Jilin 130021, China. ${ }^{2}$ Jilin Provincial Laboratory of Biomedical Engineering, Jilin University, Changchun, China. ${ }^{3}$ Center of Prosthodontics and Oral Implantology, Foshan Stomatology Hospital, School of Stomatology and Medicine, Foshan University, Foshan 528000, China. ${ }^{4}$ Center for Reproductive Medicine, Center for Prenatal Diagnosis, First Hospital, Jilin University, Changchun, China. ${ }^{5}$ Affiliated Stomatology Hospital of Guangzhou Medical University, 39 Huangsha Ave., Guangzhou 510080, Guangdong, China. ${ }^{6}$ Institute of Antler Science and Product Technology, Changchun Sci-Tech University, 1345 Pudong Rd., Changchun, Jilin 130600, China. ${ }^{7}$ Institute of Special Animal and Plant Sciences, Chinese Academy of Agricultural Sciences (CAAS), 4899 Juye St., Changchun, Jilin 130112, China.
\end{abstract}

Received: 19 February 2021 Accepted: 28 April 2021

Published online: 03 August 2021

\section{References}

1. Sorg H, Tilkorn DJ, Hager S, Hauser J, Mirastschijski U. Skin wound healing: an update on the current knowledge and concepts. Eur Surg Res. 2017; 58(1-2):81-94. https://doi.org/10.1159/000454919.

2. Reinke JM, Sorg H. Wound repair and regeneration. Eur Surg Res. 2012;49(1): 35-43. https://doi.org/10.1159/000339613.

3. Rippa AL, Kalabusheva EP, Vorotelyak EA. Regeneration of dermis: scarring and cells involved. Cells. 2019;8.

4. Lichtman MK, Otero-Vinas M, Falanga V. Transforming growth factor beta (TGF- $\beta$ ) isoforms in wound healing and fibrosis. Wound Repair Regen. 2016; 24(2):215-22. https://doi.org/10.1111/wrr.12398.

5. Kim KK, Sheppard D, Chapman HA. TGF- $\beta 1$ signaling and tissue fibrosis. Cold Spring Harb Perspect Biol. 2018;10.

6. Rosenbloom J, Macarak E, Piera-Velazquez S, Jimenez SA. Human fibrotic diseases: current challenges in fibrosis research. Methods Mol Biol. 2017; 1627:1-23

7. Wynn TA, Ramalingam TR. Mechanisms of fibrosis: therapeutic translation for fibrotic disease. Nat Med. 2012;18(7):1028-40. https://doi.org/10.1038/ nm.2807.

8. Ojeh N, Pastar I, Tomic-Canic M, Stojadinovic O. Stem cells in skin regeneration, wound healing, and their clinical Applications. Int J Mol Sci. 2015;16(10):25476-501. https://doi.org/10.3390/ijms161025476.

9. Duan M, Zhang Y, Zhang H, Meng Y, Qian M, Zhang G. Epidermal stem cellderived exosomes promote skin regeneration by downregulating transforming growth factor- $\beta 1$ in wound healing. Stem Cell Res Ther. 2020; 11(1):452. https://doi.org/10.1186/s13287-020-01971-6.

10. Zhang Y, Yan J, Liu Y, Chen Z, Li X, Tang L, Li J, Duan M, Zhang G. Human amniotic fluid stem cell-derived exosomes as a novel cell-free therapy for cutaneous regeneration. Front Cell Dev Biol. 2021;9:685873. https://doi.org/1 0.3389/fcell.2021.685873.

11. Abdelwahid E, Kalvelyte A, Stulpinas A, de Carvalho KA, Guarita-Souza LC, Foldes $\mathrm{G}$. Stem cell death and survival in heart regeneration and repair. Apoptosis. 2016;21(3):252-68. https://doi.org/10.1007/s10495-015-1203-4.
12. Rong X, Zhang G, Yang Y, Gao C, Chu W, Sun H, et al. Transplanted antler stem cells stimulated regenerative healing of radiation-induced cutaneous wounds in rats. Cell Transplant. 2020;29:963689720951549.

13. Han Y, Li X, Zhang Y, Han Y, Chang F, Ding J. Mesenchymal stem cells for regenerative medicine. Cells. 2019;8(8). https://doi.org/10.3390/cells8080886.

14. Rong $X$, Li J, Yang $Y$, Shi L, Jiang T. Human fetal skin-derived stem cell secretome enhances radiation-induced skin injury therapeutic effects by promoting angiogenesis. Stem Cell Res Ther. 2019;10(1):383. https://doi. org/10.1186/s13287-019-1456-X.

15. Lee KB, Choi J, Cho SB, Chung JY, Moon ES, Kim NS, et al. Topical embryonic stem cells enhance wound healing in diabetic rats. J Orthop Res. 2011;29(10):1554-62. https://doi.org/10.1002/jor.21385.

16. Gorecka J, Kostiuk V, Fereydooni A, Gonzalez L, Luo J, Dash B, et al. The potential and limitations of induced pluripotent stem cells to achieve wound healing. Stem Cell Res Ther. 2019;10(1):87. https://doi.org/10.1186/s13287-019-1185-1.

17. Zeinali R, Biazar E, Keshel SH, Tavirani MR, Asadipour K. Regeneration of fullthickness skin defects using umbilical cord blood stem cells loaded into modified porous scaffolds. Asaio j. 2014;60(1):106-14. https://doi.org/10.1 097/MAT.0000000000000025.

18. Doi H, Kitajima Y, Luo L, Yan C, Tateishi S, Ono Y, et al. Potency of umbilical cord blood- and Wharton's jelly-derived mesenchymal stem cells for scarless wound healing. Sci Rep. 2016;6(1):18844. https://doi.org/10.1038/srep18844.

19. Jung JA, Yoon YD, Lee HW, Kang SR, Han SK. Comparison of human umbilical cord blood-derived mesenchymal stem cells with healthy fibroblasts on wound-healing activity of diabetic fibroblasts. Int Wound J. 2018;15(1):133-9. https://doi.org/10.1111/iwj.12849.

20. Çil N, Oğuz EO, Mete E, Çetinkaya A, Mete GA. Effects of umbilical cord blood stem cells on healing factors for diabetic foot injuries. Biotech Histochem. 2017;92(1):15-28. https://doi.org/10.1080/10520295.2016.1243728.

21. Kim YJ, Yoo SM, Park HH, Lim HJ, Kim YL, Lee S, et al. Exosomes derived from human umbilical cord blood mesenchymal stem cells stimulates rejuvenation of human skin. Biochem Biophys Res Commun. 2017:493(2): 1102-8. https://doi.org/10.1016/j.bbrc.2017.09.056.

22. Baksh D, Yao R, Tuan RS. Comparison of proliferative and multilineage differentiation potential of human mesenchymal stem cells derived from umbilical cord and bone marrow. Stem Cells. 2007;25(6):1384-92. https:// doi.org/10.1634/stemcells.2006-0709.

23. Lu LL, Liu YJ, Yang SG, Zhao QJ, Wang X, Gong W, et al. Isolation and characterization of human umbilical cord mesenchymal stem cells with hematopoiesis-supportive function and other potentials. Haematologica. 2006;91(8):1017-26.

24. Chuang HM, Shih TE, Lu KY, Tsai SF, Harn HJ, Ho LI. Mesenchymal Stem Cell Therapy of Pulmonary Fibrosis: Improvement with Target Combination. Cell Transplant. 2018;27(11):1581-7. https://doi.org/10.1177/0963689718787501.

25. Samsonraj RM, Raghunath M, Nurcombe V, Hui JH, van Wijnen AJ, Cool SM. Concise review: multifaceted characterization of human mesenchymal stem cells for use in regenerative medicine. Stem Cells Transl Med. 2017;6(12): 2173-85. https://doi.org/10.1002/sctm.17-0129.

26. Pegtel DM, Gould SJ. Exosomes. Annu Rev Biochem. 2019;88(1):487-514. https://doi.org/10.1146/annurev-biochem-013118-111902.

27. Batrakova EV, Kim MS. Using exosomes, naturally-equipped nanocarriers, for drug delivery. J Control Release. 2015;219:396-405. https://doi.org/10.1016/j. jconrel.2015.07.030.

28. Meng X, Sun B, Xue M, Xu P, Hu F, Xiao Z. Comparative analysis of microRNA expression in human mesenchymal stem cells from umbilical cord and cord blood. Genomics. 2016;107(4):124-31. https://doi.org/10.101 6/j.ygeno.2016.02.006.

29. Rong X, Chu W, Zhang H, Wang Y, Qi X, Zhang G, et al. Antler stem cellconditioned medium stimulates regenerative wound healing in rats. Stem Cell Res Ther. 2019;10(1):326. https://doi.org/10.1186/s13287-019-1457-9.

30. Pardali E, Sanchez-Duffhues G, Gomez-Puerto MC, Ten Dijke P. TGF- $\beta$-induced endothelial-mesenchymal transition in fibrotic diseases. Int J Mol Sci. 2017;18.

31. Hinz B. Myofibroblasts. Exp Eye Res. 2016;142:56-70. https://doi.org/10.1016/ j.exer.2015.07.009.

32. Hu L, Wang J, Zhou X, Xiong Z, Zhao J, Yu R, et al. Exosomes derived from human adipose mensenchymal stem cells accelerates cutaneous wound healing via optimizing the characteristics of fibroblasts. Sci Rep. 2016;6(1): 32993. https://doi.org/10.1038/srep32993.

33. Samanta S, Rajasingh S, Drosos N, Zhou Z, Dawn B, Rajasingh J. Exosomes: new molecular targets of diseases. Acta Pharmacol Sin. 2018;39(4):501-13. https://doi.org/10.1038/aps.2017.162. 
34. Cai Q, Chen F, Xu F, Wang K, Zhang K, Li G, et al. Epigenetic silencing of microRNA-125b-5p promotes liver fibrosis in nonalcoholic fatty liver disease via integrin a8-mediated activation of RhoA signaling pathway. Metabolism. 2020;104:154140. https://doi.org/10.1016/j.metabol.2020.154140.

35. Sun $L, X u T$, Chen $Y, Q u$ W, Sun $D$, Song $X$, et al. Pioglitazone attenuates kidney fibrosis via miR-21-5p modulation. Life Sci. 2019;232:116609. https:// doi.org/10.1016/j.lfs.2019.116609.

36. Li Q, Li B, Li Q, Wei S, He Z, Huang X, et al. Exosomal miR-21-5p derived from gastric cancer promotes peritoneal metastasis via mesothelial-tomesenchymal transition. Cell Death Dis. 2018;9(9):854. https://doi.org/10.103 8/s41419-018-0928-8.

\section{Publisher's Note}

Springer Nature remains neutral with regard to jurisdictional claims in published maps and institutional affiliations.

Ready to submit your research? Choose BMC and benefit from:

- fast, convenient online submission

- thorough peer review by experienced researchers in your field

- rapid publication on acceptance

- support for research data, including large and complex data types

- gold Open Access which fosters wider collaboration and increased citations

- maximum visibility for your research: over $100 \mathrm{M}$ website views per year

At BMC, research is always in progress.

Learn more biomedcentral.com/submissions 